\title{
Cost-utility analysis of esketamine and electroconvulsive therapy in adults with treatment-resistant depression
}

\author{
Kinza Degerlund Maldi ${ }^{1}$, Peter Asellus ${ }^{2}$, Anna Myléus ${ }^{3}$ and Fredrik Norström ${ }^{1 *}$
}

\begin{abstract}
Background: Electroconvulsive therapy (ECT) has long been used for treating individuals with treatment-resistant depression (TRD). Esketamine has recently emerged as a new treatment for TRD due to its rapid antidepressant effects. To further inform the decision regarding choice of treatment, this paper aims to evaluate whether ECT or esketamine is the more cost-effective option.

Methods: The cost-effectiveness was derived as cost per quality-adjusted life-year (QALY) using a Markov model from a societal and life-time perspective. The incremental cost-effectiveness ratio (ICER) was calculated. Health states included different depression and remission states and death. Data to populate the model was derived from randomised controlled trials and other research. Various sensitivity analyses were carried out to test the robustness of the model.

Results: The base case scenario shows that ECT is cost-effective compared to esketamine and yields more QALYs at a lower cost. The sensitivity analysis shows that ECT is cost-effective in all scenarios and ECT dominates esketamine in 12 scenarios.

Conclusions: This study found that, from a cost-effectiveness point of view, ECT should be the first-hand option for individuals with TRD, when other first line treatments have failed. Considering the lack of economic evaluation of ECT and esketamine, this study is of great value to decision makers.
\end{abstract}

Keywords: Electroconvulsive therapy, Esketamine, Treatment-resistant depression, Cost-effectiveness, Markov model, QALY, ICER

\section{Introduction}

Major depressive disorder is one of the most prevalent and debilitating forms of mental illnesses and a major cause of morbidity worldwide. In the United Kingdom (UK), it is estimated that $6.4 \%$ of the population suffer from the condition [1]. Major depressive disorder is usually treated with antidepressant medication (AD), but some individuals do not respond to treatment and they

\footnotetext{
${ }^{*}$ Correspondence: fredrik.norstrom@umu.se

${ }^{1}$ Department of Epidemiology and Global Health, Umeå University, SE-901 87 Umeå, Sweden

Full list of author information is available at the end of the article
}

are considered suffering from treatment-resistant depression (TRD). There is no universally accepted definition of TRD, although the literature has commonly defined the condition as failure to respond to at least two trials of first line $\mathrm{AD}$ of both adequate duration and dose [2]. It is estimated that between 10 and $30 \%$ of individuals with major depressive disorder also have TRD with a significant impact on their daily life and productivity [2-5]. The societal impact of TRD is great due to its prevalence in the population, its impact on functioning, productivity and quality-of-life and its contribution to premature mortality [6]. 
Individuals with TRD can be treated with a combination of $\mathrm{AD}$, mood stabilising medication, antipsychotic medication, psychological therapy and electroconvulsive therapy (ECT). ECT is often used as a "last option" when all other treatments have failed or not been tolerated. The treatment is not routinely considered for people with moderate depression unless their depression has not responded to multiple drug and psychological treatments [3]. ECT is considered highly effective in treating TRD, with remission rates between 50 and 70\% [7]. Moreover, in a recent large observational study of older patients, ECT was associated with a lower one-year all-cause mortality and reduced suicide rates during three months after treatment [8]. Despite the consistent evidence that ECT is an effective treatment for patients with TRD, research suggest that it is underutilised [9-11]. This is partly due to limited availability of ECT, patient choice, stigma and a dated perception of the treatment [9-11]. It is also due to the fact that maintenance ECT (M-ECT) might be required, a practice that is uncommon in England [12] and not supported by UK National Institute for Health and Care Excellence (NICE) as routine treatment [13].

There has, however, been recent developments in treatments for TRD. The N-methyl-D-aspartate receptor antagonist ketamine, commonly used as a tranquiliser and pain medication, has emerged as a new treatment due to its rapid and robust antidepressant effects [14]. According to a systematic review [15], 77\% of the included studies reported significant improvement in depressive symptoms among patients receiving ketamine or esketamine compared to the control group. The review concluded that ketamine is an effective treatment option for patients with major depressive disorder when administered via intravenous, intranasal and oral routes. The review included two studies where ketamine was administered intranasal with improvement in both studies. Both the Food and Drug administration (FDA) and the European Commission have approved intranasal esketamine in combination with a selective serotonin reuptake inhibitor (SSRI) or serotonin and norepinephrine reuptake inhibitor (SNRI) for adults with TRD $[16,17]$.

In an individual with TRD, ECT and esketamine are both viable treatment options. To further inform the decision regarding choice of treatment, an economic evaluation may be utilised as it compares the difference in cost and the difference in benefits between the options. In the case of an option being dominant, costing less and generating greater benefits than the alternative, it is unequivocally cost-effective. However, if an intervention generates more health to a higher cost than the option, it can still be cost-effective. In these situations, the incremental cost-effectiveness ratio (ICER) is calculated which demonstrates the additional cost per extra unit of health [18].
To our knowledge there is no cost-effectiveness analysis evaluating whether ECT or esketamine is the more costeffective option.

\section{Aim}

The aim of this paper is to evaluate whether ECT or esketamine is the more cost-effective option for treating TRD.

\section{Method}

To determine which option is cost-effective, the ICER is estimated by calculating the difference in cost between ECT and esketamine divided by the difference in qualityadjusted life years (QALYs) between ECT and esketamine. The ICER is subsequently compared to a threshold value which will determine if the intervention is costeffective [18]. The analysis is done in the context of the UK, and NICE recommends a threshold in the range of $£ 20,000$ to $£ 30,000$ per QALY gained [19]. However, NICE does not reject nor approve based on cost-effectiveness alone [20].

\section{The model}

The cost-effectiveness analysis is based on a Markov model which defines the health and treatment states and possible consequences of the interventions. The prognosis of individuals is modelled, based on a set of possible transitions between these states over a series of discrete time periods. Cycles of 30 days was used in our Markov model as the cycle length is short enough to simulate the frequency of clinical events and treatment interventions and longer cycles can introduce more bias [21]. This model structure is more flexible than other model alternatives, such as decision trees, and allows for easy incorporation of relapses and recurrences [22]. This study is targeting a population with TRD where previous treatments have resulted in a lack of improvement and the next step in treatment would be either ECT or esketamine. It is therefore assumed that individuals enter the model at 45 years of age and the model lasts for 35 years, that is until the individuals are 80 years.

The Markov model requires three types of data: transition probabilities, health utilities and cost [23]. Data for the model was gathered from various sources, see Table 1 for the input parameters used in the analyses.

The Markov model was constructed in Excel and an overview with arrows indicating possible transition patterns can be found in Fig. 1. Individuals started in "state A depression" and received either esketamine or ECT. Individuals who remitted on esketamine transitioned to "state B remission" and received esketamine for a maximum of six months. They transitioned to "state F remission" after six months, given that they were still in 
Table 1 Input parameters for the model. Costs are displayed in British pounds (GBP) 2019

\begin{tabular}{|c|c|c|c|c|}
\hline & ECT & Source & Esketamine & Source \\
\hline \multicolumn{5}{|l|}{ Transition probability } \\
\hline Depression to remission (tpA2B) and (tpC2B) & 0.696 & (28) & 0.392 & $(27)$ \\
\hline Remission to depression (tpB2C) & 0.108 & (28) & 0.0724 & $(26)$ \\
\hline Remission to depression (tpB2C) for maintenance ECT & 0.0300 & (29) & & \\
\hline \multicolumn{5}{|l|}{ Transition probability for both ECT and esketamine } \\
\hline Depression to death (tpA2G), (tpC2G) and (tpD2G) & 0.00304 & $(31,32)$ & & \\
\hline Depression to remission (tpD2E) & 0.107 & (30) & & \\
\hline Remission to depression (tpE2D) & 0.232 & (30) & & \\
\hline \multicolumn{5}{|l|}{ Remission to death (tpB2G and tpE2G) both ECT and esketamine } \\
\hline $45-49$ & 0.000180 & Lifetable & & \\
\hline $50-54$ & 0.000265 & Lifetable & & \\
\hline $55-59$ & 0.000407 & Lifetable & & \\
\hline $60-64$ & 0.000646 & Lifetable & & \\
\hline $65-69$ & 0.00100 & Lifetable & & \\
\hline $70-74$ & 0.00163 & Lifetable & & \\
\hline $75-79$ & 0.00285 & Lifetable & & \\
\hline \multicolumn{5}{|l|}{ Utility per cycle } \\
\hline Remission & 0.07 & (33) & & \\
\hline Depression & 0.05 & (33) & & \\
\hline \multicolumn{5}{|l|}{ Cost per state and cycle age $<65$ (age $\geq 65)$} \\
\hline State $A$ and $C$ & $7932(6321)$ & & $7210(5599)$ & \\
\hline State B, standard treatment after ECT & $701(118)$ & & - & \\
\hline State $\mathrm{B}$, month $1 \mathrm{M}$-ECT and esketamine & $3313(2730)$ & & $2882(2299)$ & \\
\hline $\begin{array}{l}\text { State B, month } 2 \text { and following months M-ECT and month 2-5 } \\
\text { esketamine }\end{array}$ & $2161(1578)$ & & $1583(1000)$ & \\
\hline State B, month 6 esketamine & - & & $1890(1307)$ & \\
\hline State D & $2729(1119)$ & & $2729(1119)$ & \\
\hline State $\mathrm{E}$ and $\mathrm{F}$ & $701(118)$ & & $701(118)$ & \\
\hline
\end{tabular}

${ }^{a} \mathrm{tp}=$ transition probability, that is, the probability of the individual moving from one state to the other. tpA2B indicates the probability of the individual transitioning from state $A$ (depression) to state B (remission)

remission, and received standard treatment. If the individual relapsed while receiving esketamine in "state B remission", they transitioned to "state $C$ depression" and received esketamine at a higher dose. The transition pattern from esketamine was based on the treatment protocol by Janssen [24].

Individuals who remitted on ECT transitioned to "state $B$ remission" and received standard treatment. If the individual relapsed, they transitioned to "state $\mathrm{C}$ depression" and received ECT. Individuals who did not remit on ECT or esketamine transitioned to "state D depression" and in the case of treatment only received standard treatment. Individuals who remitted in "state D depression" transitioned to "state E remission".

An additional model was constructed where individuals received M-ECT. In this model individuals who remitted on ECT and transitioned to "state B remission" received $\mathrm{M}$-ECT. If they relapse during M-ECT they transitioned to "state C depression" and received ECT.

\section{Interventions}

\section{Standard treatment}

Individuals received $\mathrm{AD}$ in all states and visited their general practitioner (GP) once a month for this [25].

\section{Esketamine}

The treatment regimen was based on the treatment protocol by Janssen [24]. In depression (state A and C) the treatment consisted of two doses a week at a community mental health centre (CMHT). The starting dose was $56 \mathrm{mg}$, followed by a dose of $56 \mathrm{mg}$ or $84 \mathrm{mg}$ [24]. Esketamine was prescribed by a psychiatrist and administered by a nurse. Individuals continued to receive esketamine from a nurse at the CMHT, but at a lower frequency, in remission. The maintenance dose of esketamine was recommended at 56 or $84 \mathrm{mg}$ once a week the first four weeks followed by a further reduction to one dose every other week, for a maximum of six months. According to the literature, around half of the individuals received the 


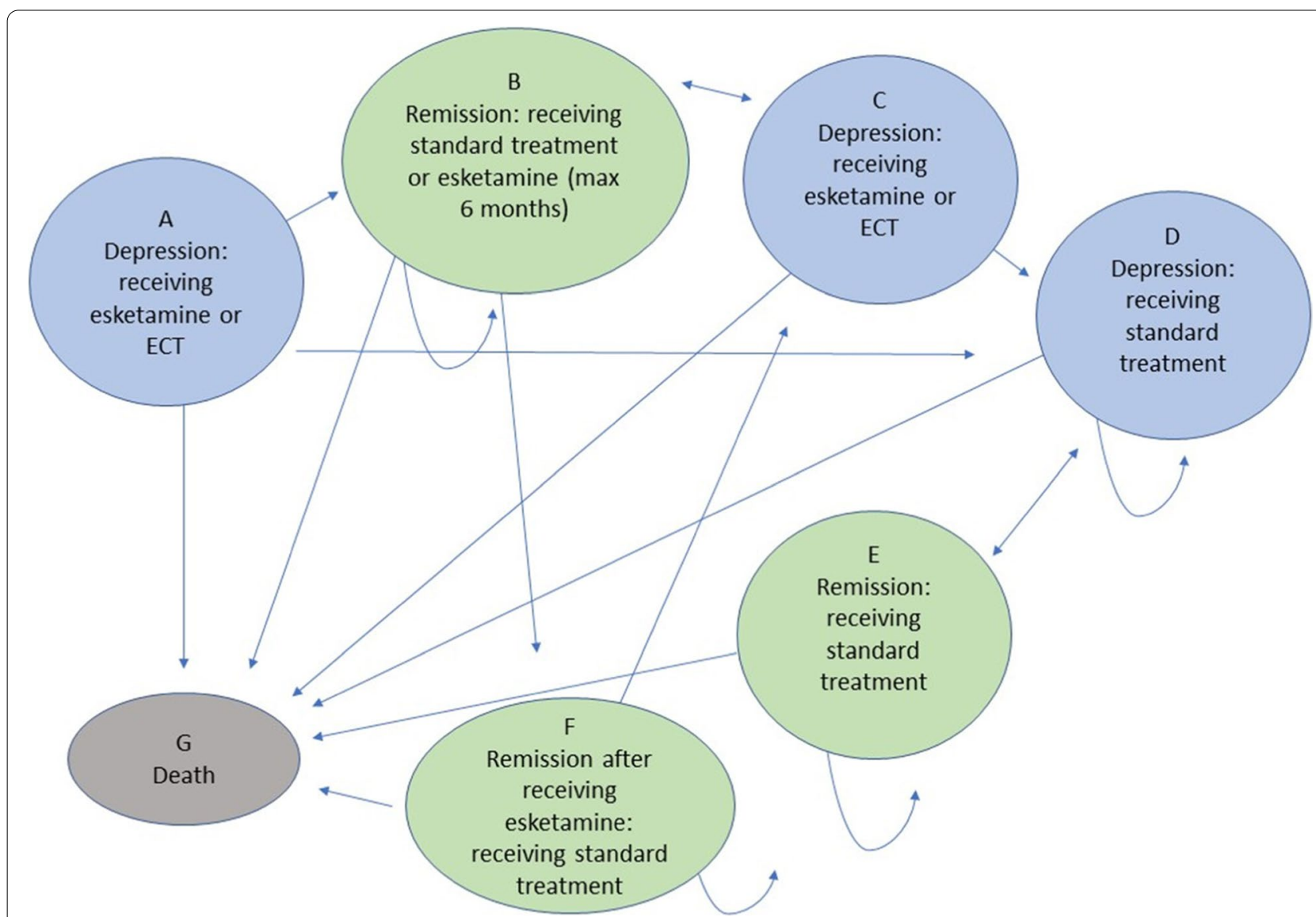

Fig. 1 Markov model schematic with the states and transition patterns. All individuals started in state A as depressed and received either electroconvulsive therapy (ECT) or esketamine. The possible transitions between the states are illustrated with arrows. Blue states indicate ongoing depression and green states indicate remission

higher dose and half the lower [26, 27], thus, an average dose was used. For example, for the first four weeks the total dose for an individual receiving $56 \mathrm{mg}$ was $448 \mathrm{mg}$ and the total dose for an individual receiving $84 \mathrm{mg}$ was $644 \mathrm{mg}$, resulting in an average dose of $546 \mathrm{mg}$. The individual had two psychiatrist appointments, one in the beginning of the cycle to be prescribed esketamine and one in the end for follow-up.

\section{ECT}

According to NICE, ECT is usually given twice a week, thus in depression (state $\mathrm{A}$ and $\mathrm{C}$ ) the individual had eight sessions of ECT and two psychiatrist appointment, one in the beginning of the cycle to be prescribed ECT and one at the end of the cycle for follow-up [13]. The model was based on one cycle of ECT in the depression state and standard treatment in remission. In the main model, ECT is not given as maintenance treatment as this appears to be fairly uncommon in the UK, with about $9.6 \%$ of individuals receiving ECT continuing with M-ECT [12]. Moreover, NICE reports little evidence regarding the value of M-ECT. However, to make the study more applicable to countries where M-ECT is used, a maintenance model with M-ECT was constructed. Individuals who remitted on ECT continued to receive M-ECT weekly for six weeks followed by twice a month.

\section{Transition probabilities}

The Markov model was populated with transition probabilities derived from various studies and converted to monthly probabilities by using eq. 1 and 2 .

$$
\begin{aligned}
& r=-\frac{[\ln (1-P)]}{t} \\
& p=1-\exp \{-r t\}
\end{aligned}
$$

Where $r=$ rate, $p=$ probability, $t=$ time period of interest.

The transition probability of remitting on esketamine was derived from a randomised, double-blind, activecontrolled study where 342 patients from Europe, North 
America and Brazil were randomised to either placebo or twice a week intranasal esketamine 56 or $84 \mathrm{mg}$ plus an $\mathrm{AD}$ [27]. Remission rates in this study were $36.0 \%$ for the $56 \mathrm{mg}$ group and $38.8 \%$ for the $84 \mathrm{mg}$ group during four weeks.

The transition probability of remitting on ECT and relapsing while receiving $\mathrm{AD}$ after successful ECT was derived from a randomised controlled trial. Out of 206 eligible patients from London UK, 46 entered the study of which 22 received ECT. Remitters were followed up for six month to capture relapse [28]. Remission from ECT was $59.1 \%$ during the study and relapse after six months was $50 \%$. For the maintenance model with M-ECT, the transition probability of relapsing while receiving M-ECT was derived from a Swedish randomised controlled trial. Out of 200 eligible patients from four different hospitals in Sweden, 56 were randomised to either receive M-ECT and pharmacotherapy or pharmacotherapy [29]. During the one year follow up, $31 \%$ of the medication resistant individuals receiving M-ECT experienced a relapse.

The transition probability of relapsing while receiving esketamine maintenance treatment was derived from a double-blind, randomised clinical trial evaluating the long-term use of esketamine [26]. Out of 1097 eligible patients from Europe, North America and Brazil, 297 were randomised to either receive esketamine or placebo. During the 17.7 weeks maintenance phase $26.7 \%$ individuals experienced a relapse.

The maintenance phase of esketamine was maximum six months and individuals transitioned to standard treatment after six months [24]. The transition probability of moving to this state was set as the same probability of remaining in remission. The transition probability of relapsing was the same as relapsing on standard treatment.

The transition probability of remitting from standard treatment and relapsing from standard treatment, was derived from the STAR*D trial. This is the largest openlabel, pragmatic trial that has been undertaken to examine the treatment of major depressive disorder [30]. In this trial, 3671 American individuals received AD medication, which was augmented and changed in those without sufficient response. The transition probability was derived from the 390 individuals who had not benefited sufficiently from two different medication trials, alike the definition of TRD. The remission rate in this study was $13.7 \%$ during 5.6 weeks and the rate of relapse was $64.6 \%$ during 3.1 months.

The transition probability of death while suffering from depression was derived from two studies. A study using a Swedish register of 118,774 individuals concluded that the increased risk of death among individuals with TRD is 1.35 times higher compared to depression [31].
This increased risk was applied to a prospective study of 20,320 individuals which estimated mortality in people with depression in the UK [32]. The study concluded that $11.6 \%$ of the study population died from all-causes over an average follow up time of 4.5 years. The transition probability of death while in remission was derived from a lifetable and calculated over five-year intervals, $45-49$ years, $50-54$ years and so on.

\section{Utility scores}

QALYs aim at capturing the health of a specific state. One year of perfect health is valued as 1 , and death is valued at 0 . Utility scores were set as 0.81 QALYs for remission and 0.57 QALYs for depression, and converted to monthy utility scores. The utility scores originates from a longitudinal study using the validated EQ-5D questionnaire to investigate the quality of life in individuals with depression in Sweden [33]. The utility scores have been used in at least two studies similar to ours [34, 35].

\section{Costing}

The study adopts a societal perspective by including direct costs related to the utilisation of medical resources, such as healthcare visits, and indirect costs related to the loss of productivity and informal care [36-38]. In contrast, a healthcare perspective only includes the direct costs. It has been argued that the loss of productivity is especially important in mood disorders as it accounts for a large proportion of the total cost burden. Individuals suffering from mood disorders lose, on average, more work days compared to individuals suffering from other chronic conditions [39].

If wages are used to quantify productivity loss, health will be valued higher for high-income earners and men, over that of low-income earners and women. For equity reasons the proposed solution is to apply a general wage rate, that is, the average wage of the country and payroll $\operatorname{tax}$ [39]. Therefore, the average full-time wage of $£ 2340$, part-time wage of $£ 680$ and payroll tax of $12 \%$ (applied to income between $£ 702.01$ and $£ 3863$ per month) were used for this study [40]. Calculations were performed under the assumption of employment until age 65 years, which is the general retirement age in the UK. After retirement ( $\geq 65$ years of age) no productivity loss was assumed. When necessary, costs were converted to 2019 years value pound sterling (GBP) by the Bank of England inflation calculator. Cost for each state is displayed in Table 1.

\section{Direct costs}

Most of the costs for TRD were taken from Table 2 in a study by McCrone et al. [25], apart from the cost for GP, psychiatrist and nurse visits, which was taken from 
Table 2 Cost-effectiveness of ECT and esketamine main model - base case and sensitivity analysis

\begin{tabular}{|c|c|c|c|c|c|c|c|}
\hline & \multicolumn{2}{|l|}{ ECT } & \multicolumn{2}{|c|}{ Esketamine } & \multicolumn{2}{|c|}{ Incremental } & \multirow[t]{2}{*}{ ICER } \\
\hline & Cost & QALY & Cost & QALY & Cost & QALY & \\
\hline \multicolumn{8}{|l|}{ Base case } \\
\hline Societal perspective & 453,693 & 14.85 & 456,211 & 14.26 & -2517 & 0.59 & $\mathrm{ECT}_{\text {dominates }}^{\mathrm{a}}$ \\
\hline Healthcare perspective & 124,530 & 14.85 & 120,390 & 14.26 & 4140 & 0.59 & 6969 \\
\hline \multicolumn{8}{|l|}{ Sensitivity analysis } \\
\hline (1a) Doubling the productivity loss & 681,736 & 14.85 & 688,301 & 14.26 & -6565 & 0.59 & ECT dominates \\
\hline (1b) Halving the productivity loss & 339,673 & 14.85 & 340,165 & 14.26 & -493 & 0.59 & ECT dominates \\
\hline (2a) ECT six times per cycle - societal & 450,071 & 14.85 & 456,211 & 14.26 & -6140 & 0.59 & ECT dominates \\
\hline (2b) ECT six times per cycle - healthcare & 121,026 & 14.85 & 120,390 & 14.26 & 636 & 0.59 & 1070 \\
\hline (2c) ECT 12 times per cycle - societal & 460,939 & 14.85 & 456,211 & 14.26 & 4728 & 0.59 & 7959 \\
\hline (2d) ECT 12 times per cycle - healthcare & 131,539 & 14.85 & 120,390 & 14.26 & 11,149 & 0.59 & 18,768 \\
\hline (3a) Increasing the remission rate from esketamine to 0.5 - societal & 453,694 & 14.85 & 458,449 & 14.36 & -4755 & 0.49 & ECT dominates \\
\hline (3b) Increasing the remission rate from esketamine to 0.5 - healthcare & 124,530 & 14.85 & 123,583 & 14.36 & 947 & 0.49 & 1927 \\
\hline (4a) Decreasing the remission rate from ECT to 0.5 - societal & 453,364 & 14.42 & 456,211 & 14.26 & -2847 & 0.16 & ECT dominates \\
\hline (4b) Decreasing the remission rate from ECT to 0.5 - healthcare & 119,350 & 14.42 & 120,390 & 14.26 & -1040 & 0.16 & ECT dominates \\
\hline $\begin{array}{l}\text { (5a) Increasing the remission rate the } 2 \text { nd time the individuals received } \\
\text { ECT/esketamine } 0.9 \text { - societal }\end{array}$ & 448,644 & 16.15 & 472,803 & 15.08 & $-24,159$ & 1.08 & ECT dominates \\
\hline $\begin{array}{l}\text { (5b) Increasing the remission rate the } 2 \text { nd time the individuals received } \\
\text { ECT/esketamine } 0.9 \text { - healthcare }\end{array}$ & 141,026 & 16.15 & 148,069 & 15.08 & -7044 & 1.08 & ECT dominates \\
\hline (6a) Five-year time horizon - societal & 105,078 & 3.00 & 116,086 & 2.81 & $-11,008$ & 0.19 & ECT dominates \\
\hline (6b) Five-year time horizon - healthcare & 31,691 & 3.00 & 31,284 & 2.81 & 406 & 0.19 & 2086 \\
\hline $\begin{array}{l}\text { (7a) Lowering QALYS (0.3) for ECT treatment during depression and increas- } \\
\text { ing QALYS ( } 0.85 \text { ) for remission after esketamine and AD - societal }\end{array}$ & 453,694 & 15.04 & 456,211 & 14.54 & -2517 & 0.5 & ECT dominates \\
\hline $\begin{array}{l}\text { (7b) Lowering QALYs (0.3) for ECT treatment during depression and increas- } \\
\text { ing QALYS (0.85) for remission after esketamine and AD - healthcare }\end{array}$ & 124,530 & 15.04 & 120,390 & 14.54 & 4140 & 0.5 & 8296 \\
\hline $\begin{array}{l}\text { (8a) Esketamine was given as long as the individual was in remission - } \\
\text { societal }\end{array}$ & 453,694 & 14.85 & 458,749 & 14.41 & -5055 & 0.45 & ECT dominates \\
\hline $\begin{array}{l}\text { (8b) Esketamine was given as long as the individual was in remission - } \\
\text { healthcare }\end{array}$ & 124,530 & 14.85 & 124,358 & 14.41 & 172 & 0.45 & 387 \\
\hline (9a) No discounting applied - societal & 466,497 & 15.22 & 469,603 & 14.60 & -3106 & 0.61 & ECT dominates \\
\hline (9b) No discounting applied - healthcare & 127,589 & 15.22 & 123,191 & 14.60 & 4399 & 0.61 & 7156 \\
\hline
\end{tabular}

${ }^{a}$ Dominates = more QALY at a lower cost

Costs are displayed in British pounds (GBP) 2019

another source [41]. The cost for ECT was derived from NICE which has estimated one session to cost $£ 558$ [13] and the cost for esketamine was derived from Jansen at $£ 163 / 28 \mathrm{mg}$ [24].

\section{Indirect costs}

Loss of productivity was weighted to account for $54 \%$ of the population being unemployed and $13 \%$ working part-time [25] while in the depression state and for $23 \%[23,25]$ of the individuals being unemployed in the remission state. These figures have been used elsewhere in a similar study [23]. The usage of informal care was derived from McCrone et al. [25] and the cost from the Office of National Statistics by applying the mean hourly wage [40]. Lastly, individuals travel to the CMHT appointment was assumed to be the similar to the London daily cap of $£ 18.80$ even if individuals are outside of London [42]. Yearly discounting of $3.5 \%$ was applied to both cost and health.

\section{Sensitivity analysis}

Sensitivity analyses were performed by alternating one key parameter at a time in the model. The study has adopted a societal perspective, but where applicable, the ICER from a healthcare perspective was also estimated.

1. Doubling and halving productivity loss.

2. Changing the frequency of ECT to six and 12 times per cycle.

3. Increasing the remission rate from esketamine to 0.5 .

4. Decreasing the remission rate from ECT to 0.5. 
5. Increasing the remission rate the second time individuals received ECT/esketamine in the depression state to 0.9 .

6. Applying a five-year time horizon.

7. Lowering QALYs to 0.3 during ECT treatment while depressed and increasing QALYs to 0.85 for remission after esketamine and after AD.

8. Esketamine was given as long as the individual was in remission, that is, longer that six months.

9. Applying no discounting for both costs and utility.

\section{Results}

Table 2 presents the results from the main model base case and the sensitivity analysis. The results show that from a societal perspective ECT dominates esketamine, that is, ECT generates more QALYs at a lower cost. From a healthcare perspective, the ICER, comparing ECT to esketamine is $£ 6969$ per QALY gained, i.e. ECT is more efficient and costlier. The ICER is below the lower border of the suggested threshold from NICE. The sensitivity analyses confirm these findings. In all scenarios ECT appears cost-effective compared to esketamine and in 12 scenarios ECT dominates esketamine, see Table 2.

Table 3 presents the results from the maintenance model with M-ECT. The base case from a societal perspective indicates that ECT followed by M-ECT is cost-effective compared with esketamine. The ICER is $£ 27,070$ per QALY gained. From a healthcare perspective the ICER is $£ 38,922$ and is thus above the suggested threshold from NICE ranging from $£ 20,000$ to $£ 30,000$ per QALY gained. The sensitivity analyses of the maintenance model indicates that ECT followed by M-ECT is cost-effective from a societal perspective in all sensitivity analyses but three, see Table 3 . Firstly, the ICER from sensitivity analysis $1 \mathrm{~b}$, halving the productivity loss, is

Table 3 Cost-effectiveness of ECT and esketamine maintenance model with M-ECT - base case and sensitivity analysis

\begin{tabular}{|c|c|c|c|c|c|c|c|}
\hline & \multicolumn{2}{|l|}{ ECT } & \multicolumn{2}{|c|}{ Esketamine } & \multicolumn{2}{|c|}{ Incremental } & \multirow[t]{2}{*}{ ICER } \\
\hline & Cost & QALY & Cost & QALY & Cost & QALY & \\
\hline \multicolumn{8}{|l|}{ Base case } \\
\hline Societal perspective & 525,707 & 16.83 & 456,219 & 14.26 & 69,488 & 2.57 & 27,070 \\
\hline Healthcare perspective & 220,303 & 16.83 & 120,392 & 14.26 & 99,911 & 2.57 & 38,922 \\
\hline \multicolumn{8}{|l|}{ Sensitivity analysis } \\
\hline (1a) Doubling the productivity loss & 736,918 & 16.83 & 688,314 & 14.26 & 48,604 & 2.57 & 18,935 \\
\hline (1b) Halving the productivity loss & 420,102 & 16.83 & 340,172 & 14.26 & 79,930 & 2.57 & 31,138 \\
\hline (2a) ECT six times per cycle during depression - societal & 522,167 & 16.83 & 456,219 & 14.26 & 65,948 & 2.57 & 25,691 \\
\hline (2b) ECT six times per cycle during depression - healthcare & 216,879 & 16.83 & 120,392 & 14.26 & 96,487 & 2.57 & 37,588 \\
\hline (2c) ECT 12 times per cycle during depression - societal & 532,787 & 16.83 & 456,219 & 14.26 & 76,568 & 2.57 & 29,828 \\
\hline (2d) ECT 12 times per cycle during depression - healthcare & 227,152 & 16.83 & 120,392 & 14.26 & 106,760 & 2.57 & 41,590 \\
\hline (3a) Increasing the remission rate from esketamine to 0.5 - societal & 525,707 & 16.83 & 458,462 & 14.36 & 67,245 & 2.46 & 27,290 \\
\hline (3b) Increasing the remission rate from esketamine to 0.5 - healthcare & 220,303 & 16.83 & 123,587 & 14.36 & 96,717 & 2.46 & 39,250 \\
\hline (4a) Decreasing the remission rate from ECT to 0.5 - societal & 490,004 & 15.54 & 456,219 & 14.26 & 33,784 & 1.28 & 26,326 \\
\hline (4b) Decreasing the remission rate from ECT to 0.5 - healthcare & 164,091 & 15.54 & 120,392 & 14.26 & 43,699 & 1.28 & 34,052 \\
\hline $\begin{array}{l}\text { (5a) Increasing the remission rate the } 2 \text { nd time the individuals received ECT/esketamine } \\
0.9 \text { - societal }\end{array}$ & 595,181 & 18.89 & 472,830 & 15.08 & 122,351 & 3.82 & 32,059 \\
\hline $\begin{array}{l}\text { (5b) Increasing the remission rate the } 2 \text { nd time the individuals received ECT/ } \\
\text { esketamine } 0.9 \text { - healthcare }\end{array}$ & 332,551 & 18.89 & 148,080 & 15.08 & 184,471 & 3.82 & 48,336 \\
\hline (6a) Five-year time horizon - societal & 127,153 & 3.21 & 116,088 & 2.81 & 11,066 & 0.4 & 27,570 \\
\hline (6b) Five-year time horizon - healthcare & 65,956 & 3.21 & 31,285 & 2.81 & 34,671 & 0.4 & 86,383 \\
\hline $\begin{array}{l}\text { (7a) Lowering QALYS (0.3) for ECT treatment during depression and increasing QALYS } \\
\text { (0.85) for remission after esketamine and AD - societal }\end{array}$ & 525,707 & 16.99 & 456,219 & 14.55 & 69,488 & 2.45 & 28,378 \\
\hline $\begin{array}{l}\text { (7b) Lowering QALYs }(0.3) \text { for ECT treatment during depression and increasing QALYs } \\
(0.85) \text { for remission after esketamine and AD - healthcare }\end{array}$ & 220,303 & 16.99 & 120,392 & 14.55 & 99,911 & 2.45 & 40,802 \\
\hline (8a) Esketamine was given as long as the individual was in remission - societal & 525,707 & 16.83 & 424,948 & 14.41 & 100,759 & 2.42 & 41,660 \\
\hline (8b) Esketamine was given as long as the individual was in remission - healthcare & 220,303 & 16.83 & 124,358 & 14.41 & 95,945 & 2.42 & 39,670 \\
\hline (9a) No discounting applied - societal & 540,562 & 17.22 & 469,612 & 14.60 & 70,950 & 2.42 & 27,063 \\
\hline (9b) No discounting applied - healthcare & 227,209 & 17.22 & 123,193 & 14.60 & 104,016 & 2.42 & 39,676 \\
\hline
\end{tabular}

Costs are displayed in British pounds (GBP) 2019 
$£ 31,138$ which is above the threshold. Secondly, the ICER from sensitivity analysis 5 , where the remission rate is increased the second time the individual received ECT or esketamine, is $£ 32,059$ which is above the threshold. Thirdly, the ICER from sensitivity analysis 8 , where the individual received esketamine as long as they were in remission, was $£ 41,660$ also above the threshold. From a healthcare perspective the estimated ICERs of the maintenance model were above the threshold in all analysis.

\section{Discussion}

This is, to our knowledge, the first study that evaluates whether ECT or esketamine is to recommend from a cost-effectiveness perspective among individuals with TRD. The base case from the main model indicated that ECT is cost-effective compared with esketamine. The results show that the absolute cost of ECT is slightly lower than the cost for esketamine and ECT generates more QALYs. Results from the sensitivity analyses demonstrate the robustness of these findings. There were some changes to the ICER, but all the sensitivity analyses resulted in ECT being cost-effective compared with esketamine. The results from the maintenance model with M-ECT somewhat supports these finding.

A few estimates in the sensitivity analysis from the main model are particularly noteworthy. Analysis number 7 (lowering QALYs for ECT, increasing QALYs for remission with esketamine and $A D$ ) still generated more QALYs for ECT than esketamine. This is due to the higher remission from ECT resulting in more QALYs despite reducing QALYs during ECT treatment. This is an important finding as studies have reported lower QALYs from ECT than from other treatments. QALYs from ECT treatment have been reported as low as 0.3 and $0.56[22,43]$ while QALYs in remission after AD has been reported as high as $0.85[44,45]$. Nguyen and Gordon [46] have reported a higher disutility from ECT at 0.104 compared with AD at 0.066 . Alike, research regarding QALYs in schizophrenia has suggested different utility scores depending on side effects from the medication [47]. For individuals experiencing difficult side effects or stigma from ECT [48], it is possible that the QALYs would differ between ECT and esketamine. Our sensitivity analysis from both models supports the fact that ECT would still be cost-effective.

Sensitivity analysis 6a (five-year time horizon) and 8a (esketamine in remission) are interesting findings. In the main model, ECT dominates esketamine in both scenarios, indicating that the time horizon which esketamine is administered does not change the cost-effectiveness. This in combination with sensitivity analysis number $5 \mathrm{a}$ and $\mathrm{b}$ (increasing the remission rate the second time the individual received esketamine to 0.9 ) suggests that administrating more esketamine, makes it less costeffective. However, the maintenance model with M-ECT yields slightly different results. In this model the ICER falls above the threshold in sensitivity analysis 5 and 8 .

The model included loss of productivity by accounting for unemployment and part-time work in the depression state and unemployment in the remission state, similarly to another cost-effectiveness analysis [23]. However, the loss of productivity did not include part-time employment in the remission state. As indicated by the sensitivity analysis, halving and doubling the productivity loss still results in ECT being cost-effective in the main model and the ICER falls just above the threshold in the maintenance model.

Other cost-effectiveness studies have assessed esketamine compared with $\mathrm{AD}$ and have concluded that esketamine is not cost-effective. Ross and Soeteman [49] compared esketamine to $\mathrm{AD}$ by using a decision-analytic model. They concluded that esketamine was likely not cost-effective and their sensitivity analysis did not find any realistic cost-effective scenarios. Agboola et al. [50] likewise evaluated the cost-effectiveness of esketamine plus background $\mathrm{AD}$ compared with $\mathrm{AD}$ alone in patients with TRD and reached similar conclusions. The current study is a valuable complement to these studies, as we now have evidence regarding ECT being cost-effective compared with esketamine.

ECT is cost-effective due to its higher remission rate, compared to esketamine, at a slightly lower cost in the main model. The remission rate for ECT was 69.6 and $50 \%$ in the sensitivity analysis while the study used for esketamine remission rate reported between 36 and $38.8 \%$. Critique towards a relaxed inclusion of participants and a relaxed definition of TRD in the esketamine trials have been voiced [51]. The trials only required individuals to have two failed treatments of any two $\mathrm{AD}$, enabling inclusion of patients in whom only SSRIs had failed. In the study by Fedgchin et al. [27] only 30.1 and $48.2 \%$ had three or more previous AD and in the study by Daly, et al. [26] 21.1 and $26.2 \%$ had tried more than two AD. Thus, clinicians might find that esketamine demonstrates less efficacy among real-world patients with higher levels of treatment resistance. The trial also included a new $\mathrm{AD}$ that the individual had not tried before, making it a possibility that part of the treatment effect came from the AD. The treatment protocol used for the maintenance phase has been evaluated and Nijs et al. [52], suggests that altering the dose of esketamine might optimise the treatment. The treatment protocol stipulates that during the maintenance phase individuals receive esketamine once a week following a further reduction to every other week. Researchers adjusted the dose for individuals relapsing during the reduction and saw that $47 \%$ improved 
by increasing the dose from bimonthly to weekly. These finding suggest that an individualised treatment plan of esketamine might optimise treatment response in the real world. However, with an increased dose comes an increased cost and potentially a higher risk for drug abuse.

The study by Eranti et al. [28] used for the transition probabilities for remission and relapse from ECT, only included 22 participants in the first phase and 16 participants in the follow-up phase. The most common reason for exclusion was not consenting to ECT and there was no statistically significant difference in mean age or sex ratio between the eligible patients who consented and those who declined to participate. Moreover, ECT was not administered for a fixed schedule, rather it was given until treatment response was evident but the mean duration was similar to the control group which was three weeks. This invites to some uncertainty regarding the transition probabilities. However, similar transition probabilities have been reported from another study [53], and the sensitivity analysis with a lower remission rate did not result in any noteworthy changes to the ICER. On the other hand, the strength of the study by Eranti et al. [28] and the study by Rush et al. [30], used for the relapse and remission rates for standard treatment, was that the same patients who remitted were followed-up for relapse, mimicking real-world conditions. Moreover, the studies used to populate the model had similar definitions of TRD. As mentioned previously, the esketamine studies had a more relaxed criteria, while the other studies applied the definition of two failed AD.

\section{Strengths and limitations}

A strength of the study is that it is one of the first of its kind, estimating the cost-effectiveness of ECT and esketamine. The study adopts a societal perspective and has included a broad range of indirect costs of TRD, rather than the cost of depression. This might result in a more accurate estimate of the actual cost of TRD. The study used a UK context with regards to costs and how the health care system is organised. Nonetheless, the findings are generalisable to contexts with similar healthcare systems and the maintenance model is applicable to countries using M-ECT.

Several limitations need to be considered in the study. Firstly, the studies used for QALYs employed EQ-5D to generate these and it has been argued that EQ-5D might not be adequately sensitive to changes in functioning and quality of life due to mental illness [54]. Secondly, the study utilised to derive the remission and relapse rates for esketamine had a low threshold for TRD. Thirdly, the models did not include disutility for side-effect as the research on QALYs during esketamine treatment is limited. Fourthly, the study did not address heterogeneity of different population characteristics. Lastly, the lack of long-term research of esketamine is a limitation as it could make the estimates unreliable. However, the sensitivity analysis using a five-year time horizon did not yield noteworthy changes to the costeffectiveness. Adding to this, randomised controlled trials were used to populate the model, which can be a limitation, as they might not reflect real-world conditions. Thus, further economic evaluations are warranted with longitudinal or naturalistic studies.

\section{Conclusion}

This study found that, from a cost-effectiveness point of view, ECT should be the first-hand option for individuals with TRD, when other first line treatments have failed. The time horizon did not change the estimates noteworthy and there was no scenario in the main model where esketamine was cost-effective. Nonetheless, esketamine could potentially be an option for individuals not able to undergo ECT. Further research regarding potential subgroups of individuals where esketamine treatment could be cost-effective is warranted. Considering the lack of economic evaluation of ECT and esketamine, this study is of great value to decision makers.

\section{Abbreviations \\ AD: Antidepressant medication; CMHT: Community mental health center; ECT: Electroconvulsive therapy; FDA: Food and Drug Administration; ICER: Incre- mental cost-effectiveness ratio; QALY: Quality-adjusted life years; SNRI: Seroto- nin and norepinephrine reuptake inhibitor; SSRI: Selective serotonin reuptake inhibitor; TP: Transition probability; TRD: Treatment resistant depression; NICE: National Institute for Health and Care Excellence; UK: United Kingdom.}

\section{Acknowledgements}

Not applicable.

\section{Authors' contributions}

KDM designed the study in collaboration with FN. KDM performed the analyses and all co-authors contributed to the interpretation of the results. KDM drafted the first version of the manuscript and all authors reviewed it actively. KDM wrote the final version of the manuscript and all authors read and approved the final version.

\section{Funding}

Open access funding provided by Umea University. None of the authors received funding for conducting the study.

Availability of data and materials

All data generated or analysed during this study are included in this published article.

\section{Declarations}

Ethics approval and consent to participate Not applicable. 


\section{Consent for publication \\ Not applicable.}

\section{Competing interests}

PA has received consulting fees from Janssen. The other authors declare no competing interests.

\section{Author details}

${ }^{1}$ Department of Epidemiology and Global Health, Umeå University, SE-901 87 Umeå, Sweden. ²Department of Clinical Sciences, Division of Psychiatry, Umeå University, Umeå, Sweden. ${ }^{3}$ Department of Public Health and Clinical Medicine, Family Medicine, Umeå University, Umeå, Sweden.

\section{Received: 27 June 2021 Accepted: 11 November 2021} Published online: 07 December 2021

\section{References}

1. Smith DJ, Nicholl BI, Cullen B, Martin D, Ul-Haq Z, Evans J, et al. Prevalence and characteristics of probable major depression and bipolar disorder within UK biobank: cross-sectional study of 172,751 participants. PLoS One. 2013;8(11):e75362.

2. Feifel D, Malcolm B, Boggie D, Lee K. Low-dose ketamine for treatment resistant depression in an academic clinical practice setting. J Affect Disord. 2017;221:283-8.

3. NICE. Depression in adults: recognition and management. 2020. https:// www.nice.org.uk/guidance/cg90. Accessed 6 Apr 2019.

4. McLachlan G. Treatment resistant depression: what are the options? Bmj. 2018;363:k5354.

5. Al-Harbi KS. Treatment-resistant depression: therapeutic trends, challenges, and future directions. Patient Prefer Adherence. 2012;6:369-88.

6. Patten SB, Lee RC. Towards a dynamic description of major depression epidemiology. Epidemiol Psichiatr Soc. 2004;13(1):21-8.

7. Greenberg RM, Kellner $\mathrm{CH}$. Electroconvulsive therapy: a selected review. Am J Geriatr Psychiatry. 2005;13(4):268-81.

8. Rhee TG, Sint K, Olfson M, Gerhard T, S HB, Wilkinson ST. Association of ECT with risks of all-cause mortality and suicide in older Medicare patients. Am J Psychiatry. 2021. https://doi.org/10.1176/appi.ajp.2021. 21040351.

9. Wilkinson ST, Agbese E, Leslie DL, Rosenheck RA. Identifying recipients of electroconvulsive therapy: data from privately insured Americans. Psychiatr Serv. 2018;69(5):542-8.

10. Wilkinson ST, Rosenheck RA. Electroconvulsive therapy at a veterans health administration medical center. J ect. 2017;33(4):249-52.

11. Rakita U, Bingham K, Fung K, Giacobbe P. Factors associated with global variability in electroconvulsive therapy utilization. J ect. 2017;33(4):253-9.

12. Royal College of Psychiatrists. ECT minimum dataset 2016-17 activity data report - England, Wales, Northern Ireland \& Republic of Ireland. Royal College of Psychiatrists. 2017. https://www.rcpsych.ac.uk/docs/ default-source/improving-care/ccqi/quality-networks/electro-convu Isive-therapy-clinics-(ectas)/ectas-dataset-report-2016-17.pdf. Accessed 20 Mar 2021.

13. NICE. Guidance on the use of electroconvulsive therapy. 2009. https:// www.nice.org.uk/quidance/ta59. Accessed 6 Apr 2019.

14. Veraart JKE, Smith-Apeldoorn SY, Spaans HP, Kamphuis J, Schoevers RA. Is ketamine an appropriate alternative to ECT for patients with treatment resistant depression? A systematic review. J Affect Disord. 2021;281:82-9.

15. Memon RI, Naveed S, Faquih AE, Fida A, Abbas N, Chaudhary AMD, et al. Effectiveness and safety of ketamine for unipolar depression: a systematic review. Psychiatr Q. 2020;91(4):1147-92.

16. Mahase E. Esketamine is approved in Europe for treating resistant major depressive disorder. BMJ. 2019; https://doi.org/10.1136/bmj.l7069.

17. FDA approves new nasal spray medication for treatment-resistant depression; available only at a certified doctor's office or clinic. 2019. https://www.fda.gov/news-events/press-announcements/fda-appro ves-new-nasal-spray-medication-treatment-resistant-depression-avail able-only-certified. Accessed 10 Apr 2019.

18. Andrew B, Karl C, Mark S. Decision modelling for health economic evaluation. Oxford: Oxford University Press; 2006.
19. Gandjour A. Willingness to pay for new medicines: a step towards narrowing the gap between NICE and IQWiG. BMC Health Serv Res. 2020:20(1):343.

20. Appleby J, Devlin N, Parkin D. NICE's cost effectiveness threshold. BMJ. 2007. https://doi.org/10.1136/bmj.39308.560069.BE.

21. Chhatwal J, Jayasuriya S, Elbasha EH. Changing cycle lengths in state-transition models: challenges and solutions. Med Decis Mak. 2016;36(8):952-64.

22. Vallejo-Torres L, Castilla I, González N, Hunter R, Serrano-Pérez P, PeresteloPérez L. Cost-effectiveness of electroconvulsive therapy compared to repetitive transcranial magnetic stimulation for treatment-resistant severe depression: a decision model. Psychol Med. 2015;45(7):1459-70.

23. Fitzgibbon KP, Plett D, Chan BCF, Hancock-Howard R, Coyte PC, Blumberger DM. Cost-utility analysis of electroconvulsive therapy and repetitive transcranial magnetic stimulation for treatment-resistant depression in Ontario. Can J Psychiatr. 2020;65(3):164-73.

24. Janssen. Spravato. 2020. http://www.janssenlabels.com/packageinsert/product-monograph/prescribing-information/SPRAVATO-pi.pdf. Accessed 04 Mar 2020.

25. McCrone P, Rost F, Koeser L, Koutoufa I, Stephanou S, Knapp M, et al. The economic cost of treatment-resistant depression in patients referred to a specialist service. J Ment Health. 2018;27(6):567-73.

26. Daly EJ, Trivedi MH, Janik A, Li H, Zhang Y, Li X, et al. Efficacy of Esketamine nasal spray plus Oral antidepressant treatment for relapse prevention in patients with treatment-resistant depression: a randomized clinical trial. JAMA Psychiatry. 2019;76(9):893-903.

27. Fedgchin M, Trivedi M, Daly EJ, Melkote R, Lane R, Lim P, et al. Efficacy and safety of fixed-dose Esketamine nasal spray combined with a new Oral antidepressant in treatment-resistant depression: results of a randomized, double-blind, active-controlled study (TRANSFORM-1). Int J Neuropsychopharmacol. 2019;22(10):616-30.

28. Eranti S, Mogg A, Pluck G, Landau S, Purvis R, Brown RG, et al. A randomized, controlled trial with 6-month follow-up of repetitive transcranial magnetic stimulation and electroconvulsive therapy for severe depression. Am J Psychiatry. 2007;164(1):73-81.

29. Nordenskjöld A, von Knorring L, Ljung T, Carlborg A, Brus O, Engström I. Continuation electroconvulsive therapy with pharmacotherapy versus pharmacotherapy alone for prevention of relapse of depression: a randomized controlled trial. J ect. 2013;29(2):86-92.

30. Rush AJ, Trivedi MH, Wisniewski SR, Nierenberg AA, Stewart JW, Warden D, et al. Acute and longer-term outcomes in depressed outpatients requiring one or several treatment steps: a STAR*D report. Am J Psychiatry. 2006;163(11):1905-17.

31. Reutfors J, Andersson TM, Brenner P, Brandt L, DiBernardo A, Li G, et al. Mortality in treatment-resistant unipolar depression: a register-based cohort study in Sweden. J Affect Disord. 2018;238:674-9.

32. Das-Munshi J, Chang CK, Schofield P, Stewart R, Prince MJ. Depression and cause-specific mortality in an ethnically diverse cohort from the UK: 8-year prospective study. Psychol Med. 2019;49(10):1639-51.

33. Sobocki P, Ekman M, Agren H, Runeson B, Jonsson B. The mission is remission: health economic consequences of achieving full remission with antidepressant treatment for depression. Int J Clin Pract. 2006:60(7):791-8

34. Groessl EJ, Tally SR, Hillery N, Maciel A, Garces JA. Cost-effectiveness of a Pharmacogenetic test to guide treatment for major depressive disorder. J Manag Care Spec Pharm. 2018;24(8):726-34.

35. Maniadakis N, Kourlaba G, Mougiakos T, Chatzimanolis I, Jonsson L. Economic evaluation of agomelatine relative to other antidepressants for treatment of major depressive disorders in Greece. BMC Health Serv Res. 2013;13:173.

36. Ten JB. Arguments for a societal perspective in the economic evaluation of medical innovations. Eur J Health Econ. 2009:10(4):357-9.

37. Hodgson TA, Meiners MR. Cost-of-illness methodology: a guide to current practices and procedures. Milbank Mem Fund Q Health Soc. 1982;60(3):429-62.

38. Eisenberg JM. Clinical economics. A guide to the economic analysis of clinical practices. Jama. 1989;262(20):2879-86.

39. Lensberg BR, Drummond MF, Danchenko N, Despiegel N, Francois C. Challenges in measuring and valuing productivity costs, and their relevance in mood disorders. Clinicoecon Outcomes Res. 2013;5:565-73. 
40. ONS. Employee earnings in the UK: 2019. 2019. https://www.ons.gov.uk/ employmentandlabourmarket/peopleinwork/earningsandworkingho urs/bulletins/annualsurveyofhoursandearnings/2019. Accessed 06 Jun 2020.

41. NHS. National Cost Collection for the NHS. 2020. https://www.england. nhs.uk/national-cost-collection/. Accessed 10 Jun 2020.

42. Tfl. Pay as you go caps. 2020. https://tfl.gov.uk/fares/find-fares/tube-andrail-fares/pay-as-you-go-caps. Accessed 06 Jun 2020.

43. Zhao YJ, Tor PC, Khoo AL, Teng M, Lim BP, Mok YM. Cost-effectiveness modeling of repetitive transcranial magnetic stimulation compared to electroconvulsive therapy for treatment-resistant depression in Singapore. Neuromodulation. 2018;21(4):376-82.

44. Young AH, Evitt L, Brignone M, Diamand F, Atsou K, Campbell R, et al. Cost-utility evaluation of vortioxetine in patients with major depressive disorder experiencing inadequate response to alternative antidepressants in the United Kingdom. J Affect Disord. 2017;218:291-8.

45. Khoo AL, Zhou HJ, Teng M, Lin L, Zhao YJ, Soh LB, et al. Network Metaanalysis and cost-effectiveness analysis of new generation antidepressants. CNS Drugs. 2015;29(8):695-712.

46. Nguyen KH, Gordon LG. Cost-effectiveness of repetitive transcranial magnetic stimulation versus antidepressant therapy for treatment-resistant depression. Value Health. 2015;18(5):597-604.

47. Lenert LA, Sturley AP, Rapaport MH, Chavez S, Mohr PE, Rupnow M. Public preferences for health states with schizophrenia and a mapping function to estimate utilities from positive and negative symptom scale scores. Schizophr Res. 2004;71(1):155-65.

48. Gazdag G, Ungvari GS. Electroconvulsive therapy: 80 years old and still going strong. World J Psychiatry. 2019;9(1):1-6.

49. Ross EL, Soeteman DI. Cost-effectiveness of Esketamine nasal spray for patients with treatment-resistant depression in the United States. Psychiatr Serv. 2020;71(10):988-97.

50. Agboola F, Atlas SJ, Touchette DR, Fazioli K, Pearson SD. The effectiveness and value of Esketamine for the Management of Treatment-Resistant Depression. J Manag Care Spec Pharm. 2020;26(1):16-20.

51. Turner EH. Esketamine for treatment-resistant depression: seven concerns about efficacy and FDA approval. Lancet Psychiatry. 2019;6(12):977-9.

52. Nijs M, Wajs E, Aluisio L, Turkoz I, Daly E, Janik A, et al. Managing Esketamine treatment frequency toward successful outcomes: analysis of phase 3 data. Int J Neuropsychopharmacol. 2020;23(7):426-33.

53. Jelovac A, Kolshus E, McLoughlin DM. Relapse following successful electroconvulsive therapy for major depression: a meta-analysis. Neuropsychopharmacology. 2013;38(12):2467-74.

54. Knapp M, Mangalore R. The trouble with QALYs. Epidemiol Psichiatr Soc. 2007;16(4):289-93.

\section{Publisher's Note}

Springer Nature remains neutral with regard to jurisdictional claims in published maps and institutional affiliations.

Ready to submit your research? Choose BMC and benefit from:

- fast, convenient online submission

- thorough peer review by experienced researchers in your field

- rapid publication on acceptance

- support for research data, including large and complex data types

- gold Open Access which fosters wider collaboration and increased citations

- maximum visibility for your research: over $100 \mathrm{M}$ website views per year

At BMC, research is always in progress.

Learn more biomedcentral.com/submissions 